\title{
Valgus extension overload syndrome of the elbow in a test cricket fast bowler
}

\author{
Mandeep S Dhillon (MS, MNAMS) ${ }^{1}$ \\ Prabhudev Prasad (MS) ${ }^{1}$ \\ Akshay Goel (MS) ${ }^{2}$ \\ Himmat S Dhillon (BPt) \\ ${ }^{1}$ Department of Orthopaedics, Post Graduate Institute of Medical Education and Research, Chandigarh, India \\ ${ }^{2}$ Government Medical College and Hospital Sector 32, Chandigarh, India \\ ${ }^{3}$ Department of Physiotherapy, Guru Nanak Dev University, Amritsar, India
}

\section{Introduction}

Elbow injury in sports is dependent on the nature of the game. Welldefined injury patterns like the valgus hyperextension syndrome have been reported in throwers, in racquet sports and in baseball. The demands of throwing in cricket theoretically do not involve significant stress on the elbow. We report for the first time a typical case of valgus hyperextension syndrome in an international cricket fast bowler. The mechanism of trauma seems to involve a terminal thrust at the elbow with stress concentration on the posterior and lateral sides and with some degree of distraction on the medial side.

\section{Case report}

A 29-year-old international Sri Lankan test cricket fast bowler was seen in 2004, with a 6-month history of left elbow pain and limited elbow extension (bowling arm). He had insidious pain and motion restriction without significant injury or swelling. Motion range was $15^{\circ}$ $-140^{\circ}$, with tenderness below and anterior to the medial epicondyle and olecranon tip. Radiographs of the elbow showed osteophytes at the postero-medial aspect and tip of the olecranon, and anteriorly at the coronoid tip (Fig. 1a, b). MR imaging confirmed osteophyte location and showed attenuation of the medial collateral ligament, with medullary oedema at the lateral side of the elbow (Fig. 2).

The patient refused arthroscopy, and opted for total abstinence from throwing and fast-bowling for 12 weeks. He started a training programme designed to strengthen his forearm flexor-pronator and triceps group of muscles. He resumed international cricket within 6 months without limitations in his bowling and throwing. He has since played in the 2007 Cricket World Cup, numerous test and one-day

\section{CORRESPONDENCE:}

Professor M S Dhillon

92, Sector 24 A, Chandigarh

India

Tel.: (91) 9815951090

Fax: (91) 1722744401

E-mail: drdhillon@gmail.com matches, and has taken more than 100 international wickets since recovering from injury.

\section{Discussion}

Overuse injuries constitute the vast majority of elbow disorders in athletes who throw as a requirement of the sport. ${ }^{3}$ Throwing motion is divided into six phases, ${ }^{1}$ namely wind-up, early cocking, late cocking, acceleration, deceleration and follow-through. All phases, except wind-up, are associated with elbow injury. The overhead throwing motion is needed in many sports like baseball pitching, tennis serve, volleyball spike and rugby pass. Baseball pitching generates large valgus/extension forces. ${ }^{2,5}$ Biomechanical testing has resulted in estimated valgus forces as high as $64 \mathrm{Nm}$ at the elbow during the late cocking and early acceleration phases of throwing with compressive forces of $500 \mathrm{~N}$ at the lateral radiocapitellar articulation as the elbow moves from approximately $110^{\circ}$ to $20^{\circ}$ of flexion, with throwing velocities as high as $3000^{\circ} . \mathrm{s}^{-1}$. $^{2,5}$ Large valgus loads with rapid elbow extension produce tensile stresses on medial compartment restraints (ulnar collateral ligament, flexor-pronator mass and ulnar nerve) and shear stress in the posterior compartment (posteromedial tip of the olecranon, trochlea, olecranon fossa) and compression stresses laterally (radial head, capitellum). ${ }^{6}$ This phenomenon has been termed 'valgus extension overload syndrome' and forms the basic pathophysiological model behind elbow injuries in the throwing athlete. ${ }^{6}$

Overuse injuries in cricketers occur predominantly in bowlers or fielders. ${ }^{4}$ There are no published data on bowlers' elbow overuse injury, although anecdotal reports exist. There are 3 distinct phases of the bowling action: the run-up, the delivery stride and the followthrough. ${ }^{4}$ In the delivery stride, the bowler winds the arm into a hyperflexed position and then brings the arm over the shoulder in full circumduction, releasing the ball at the start of the downward arc. During the delivery phase of the bowling action, the arm must be kept as straight as possible according to the laws of the game, otherwise it amounts to throwing or chucking. The bowler is required to not straighten the elbow more than $15^{\circ}$ during the delivery phase of the bowling action. This is different to baseball pitching or throwing a ball from the outfield. Thus valgus extension overload seems improbable in cricket bowling, as there is minimal elbow jerking during the terminal delivery arc.

However, for valgus extension overload in this particular bowler, we presume that some terminal jerk or arm straightening at delivery stage produced valgus/extension forces. The repetitive nature of 


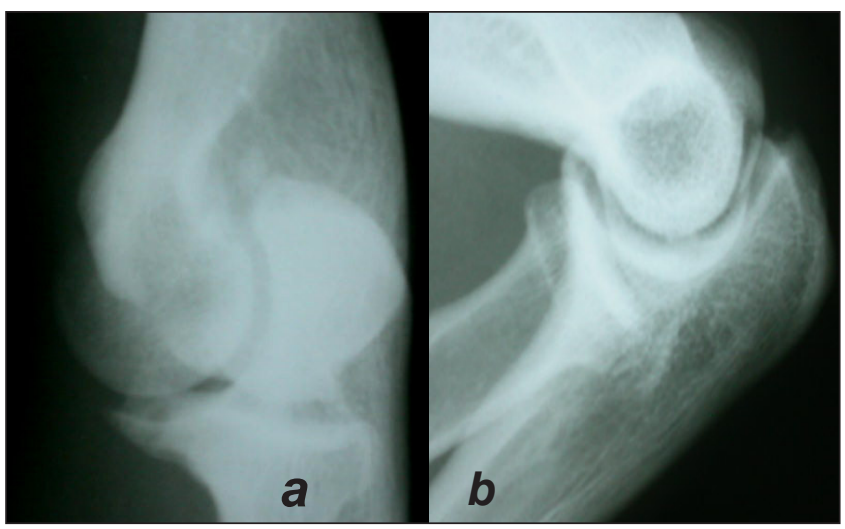

Fig. 1a and 1b. Osteophyte formation at the tip of the olecranon and tip of the coronoid.

terminal olecranon impingement at delivery could cause pressure symptoms, since the elbow is stressed into some valgus at terminal extension in fast bowlers. The other reason could be the fault in elbow position while throwing the ball during fielding. Although we were not able to ascertain the exact cause of valgus extension overload in this bowler, conservative treatment was successful and he could return to his original level of sport participation after 6 months.

Since this test bowler presented to us, the senior author has noted similar features of pain associated with throwing in other cricketers. Some test batsmen have been recorded to have similar MRI changes when clinically painful elbows were evaluated (John Orchard: personal communication). The logical cause of injury in these players is throwing during fielding, but causes related to their batting action need to be examined more carefully.

This case is reported to highlight its uniqueness and to emphasise the possibility of overload syndromes in the differential diagnosis of elbow problems in cricketers. More studies are needed to identify players with elbow pain at all levels of the sport, and in particular the relationship to age, duration of cricket career, mode of onset, player type and duration of symptoms with the goal of identifying the specific cause.

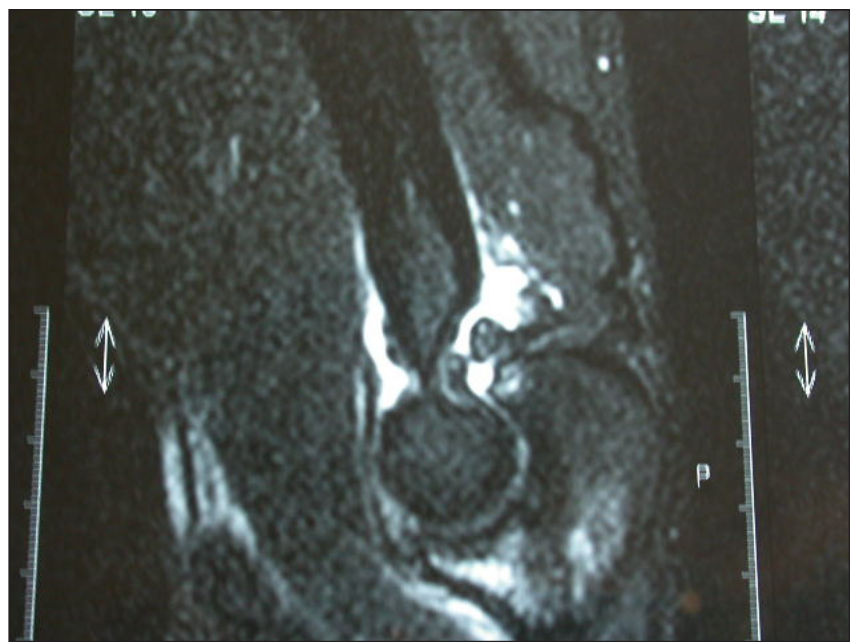

Fig. 2. MRI scan showing fluid inside the joint, osteophyte formation and signal change in the olecranon.

\section{REFERENCES}

1. Digiovane $\mathrm{M}$, Jobe $\mathrm{F}$, Pink $\mathrm{M}$, et al. An electromyographic analysis of the upper extremity in pitching. J Shoulder Elbow Surg 1992; 1: 15-25.

2. Fleisig GS, Andrews JR, Dillman CJ, Escamilla RF. Kinetics of baseball pitching with implication about injury mechanism. Am J Sports Med 1995; 23: 233-9.

3. Gerbino PG. Elbow disorders in throwing athletes. Orthop Clin N Am 2003; 34: $417-26$.

4. Myers P, O'Brien BS. Cricket injuries, rehabilitation and training. Sports Med Arthroscopy Rev 2001; 9: 124-36.

5. Werner SL, Murray TA, Hawkins RJ, Gill TJ. Relationship between throwing mechanics and elbow valgus in professional baseball pitchers. J Shoulder Elbow Surg 2002; 11: 151-5.

6. Wilson FD, Andrews JR, Blackburn TA, McCluskey G. Valgus extension overload in the pitching elbow. Am J Sports Med 1983; 11: 83-8. 